\title{
Vibrotactile intensity and frequency information in the Pacinian system: A psychophysical model
}

\author{
SLIMAN BENSMAÏA, MARK HOLLINS, and JEFFREY YAU \\ University of North Carolina, Chapel Hill, North Carolina
}

\begin{abstract}
The objective of the study was to characterize the Pacinian representation of stimulus waveform. Subjects were presented with pairs of high-frequency vibrotactile stimuli that varied in intensity and/or frequency content and made same-different judgments under conditions of low-frequency adaptation designed to minimize the contribution of the RA system. We wished to infer the nature of the information conveyed by the Pacinian system about the stimuli from measured sensitivity $\left(d^{\prime}\right)$ to stimulus differences. We first tested the hypothesis that the Pacinian system conveys only intensive information about vibratory stimuli and found that intensive cues could not account for much of the variance in the discrimination data. We then proposed a model characterizing the Pacinian-mediated representation of an arbitrary stimulus as a pattern of activation in a set of frequency-tuned minichannels. The model was shown to predict the discriminability of the stimulus pairs presented in the psychophysical experiments. Furthermore, the model parameters, optimized to fit the discrimination data, were compatible with analogous values obtained in other experimental contexts. One of the assumptions underlying the model is that information about individual spectral components is conveyed in parallel and quasi-independently. By simulating the response of a population of Pacinian afferents to a polyharmonic stimulus, we demonstrated that such a population can simultaneously convey information about multiple frequency components, despite having a homogeneous spectral profile.
\end{abstract}

The Pacinian system has been found to mediate the tactile perception of high-frequency vibrations (Bolanowski, Gescheider, Verrillo, \& Checkosky, 1988; Horch, 1991; Hyvärinen, Sakata, Talbot, \& Mountcastle, 1968; Mountcastle, Talbot, Darian-Smith, \& Kornhuber, 1967; Mountcastle, Talbot, Sakata, \& Hyvärinen, 1969; Ochoa \& Torebjörk, 1983; Talbot, Darian-Smith, Kornhuber, \& Mountcastle, 1968; Verrillo, 1966). However, despite the abundance of studies on this sensory channel, few have investigated its ability to convey information about nonsinusoidal waveforms (see Bensmaïa \& Hollins, 2000; Formby, Morgan, Forrest, \& Raney, 1992; Horch, 1991; Lamoré, Muijser, \& Keemink, 1986; Morley, Archer, Ferrington, Rowe, \& Turman, 1990; Weisenberger, 1986). In a previous study (Bensmaïa \& Hollins, 2000), we found that subjects could not use Pacinian signals to discriminate between diharmonic stimuli that differed only in the phase angle of the high-frequency component with respect to the low-frequency component. The fact that phase relations among frequency components are ignored suggests that spectral composition, rather than waveform per se, plays a primary role in shaping the Pacinian representation of a complex wave. Beyond that, the degree to which

This work was supported by the Cognitive Science Program of the University of North Carolina at Chapel Hill. We thank Kerry Ledoux for careful reading of the manuscript. Correspondence concerning this article should be addressed to S. Bensmaïa, Mind/Brain Institute, Johns Hopkins University, 3400 N. Charles St., Baltimore, MD 21218 (email: sliman@mbi.mb.jhu.edu). the Pacinian system can convey information about complex vibratory stimuli is as yet unspecified.

Even with regard to the discriminability of sinusoidal waveforms within the Pacinian range, the literature is inconclusive. An early study by Goff (1967) showed frequency discrimination to be rather poor and to grow worse (i.e., Weber fractions increased) as the standard frequency increased; once vibrotactile channel specificity had been established (Verrillo, 1968), Goff's results seemed to suggest that the Pacinian channel, when isolated, was capable of mediating only rudimentary frequency discrimination. In later work, using improved stimulus control, the Weber fraction was found to remain roughly constant across the high-frequency portion of the spectrum (Mountcastle, Steinmetz, \& Romo, 1990; Mountcastle et al., 1969). Perhaps the most remarkable report of frequency discrimination was that of Franzén and Nordmark (1975), who found Weber fractions as low as 0.03 at frequencies up to $384 \mathrm{~Hz}$; but their stimuli were brief, half-wave rectified blips, rather than sinusoids.

In the present study, we measured subjects' ability to distinguish between pairs of high-frequency simple and polyharmonic stimuli. The objective was to infer, from the pattern of confusions, the degree to which the Pacinian system could convey temporal and intensive information about the stimuli. We wished to ascertain whether the discriminability of a pair of stimuli could be fully explained in terms of the intensity of these stimuli, as previous findings might suggest (Makous, Friedman, \& Vierck, 1995), or whether temporal factors also play a role in shaping the vibratory percept. We first imple- 
mented a psychophysical model — that is, a model relating a stimulus to its sensory representation -in an attempt to account for the discrimination data solely in terms of spectral power. We concluded that subjects' performance could not be predicted on the basis of intensity cues alone. Accordingly, we sought to develop a framework describing how temporal and intensive cues combine to yield the observed pattern of confusions.

The Pacinian system has been likened to a critical band filter, drawing an analogy with the auditory system (Makous et al., 1995; Marks, 1979). This analogy is apt, given the similarity of the relevant energies in the two sensory modalities. Taking the auditory analogy one step further, we wished to assess the extent to which vibrotactile representations are functionally analogous to their auditory counterparts. To that end, we drew from theories describing the representation of pure tones in the auditory system (Moore \& Patterson, 1986) and from theories describing the representation of contrast gratings in the visual system (DeValois \& DeValois, 1988) to develop a model that describes the informational content of the Pacinian representations of simple and complex vibrotactile stimuli. The model proposes that stimuli differing in their temporal and intensive characteristics produce different patterns of activation in a set of frequency-tuned minichannels - analogous to spatial frequency channels described for the visual system (DeValois \& DeValois, 1988) - and that the perceived dissimilarity of pairs of stimuli is a function of differences in the patterns of activation they elicit in these hypothetical channels. The model incorporates assumptions that (1) the Weber fraction for frequency discrimination is constant across frequencies, (2) the subjective intensity of a given spectral component is a power function of its Pacinian-weighted power, and (3) individual components of polyharmonic stimuli are represented quasi-independently within the Pacinian system.

Since there is no evidence to suggest that individual Pacinian neurons in the somatosensory cortex are narrowly tuned to stimulus frequency (Ferrington \& Rowe, 1980; Mountcastle et al., 1969), we remain noncommittal as to the neural substrates underlying the representation of stimulus frequency and amplitude. However, by simulating the response of a population of Pacinian receptors to a polyharmonic stimulus, we explore the possibility that such a population can convey information about multiple frequency components simultaneously, despite having a uniform spectral profile.

\section{METHOD}

\section{Subjects}

The subjects were 4 male and 2 female students from the University of North Carolina, ranging in age from 22 to 31 years. Four of the 6 participated in both sets of discriminations making up the study. A 5 th subject discontinued his participation after completing the first set of discriminations and was replaced by one of the authors (J.Y.) for the second set. Except for J.Y., all the subjects were paid for their participation and were naive as to the purposes of the experiments. J.Y.'s data closely resembled those of the other subjects.

\section{Apparatus and Stimuli}

Vibrotactile stimuli were delivered through a vibrator (Minishaker 4810, Brüel \& Kjær), mounted on an adjustable platform under the experimental table. A circular Delrin contactor with a 1-cm diameter was fixed to the vibrator and passed through a hole in the table. The hole was fitted with a Plexiglas annulus with an inner diameter of $1.2 \mathrm{~cm}$, which left a 1-mm gap between contactor and surround. The vibrator was driven by an arbitrary waveform generator (PCIP-AWFG, Keithley) via a power amplifier (Type 2706, Brüel \& Kjær). Stimulus waveform and intensity were monitored via an accelerometer (Type 4371, Brüel \& Kjær), whose signal was amplified (charge amplifier, Type 2635, Brüel \& Kjær) and was sampled at $50 \mathrm{kHz}$ by an analog-to-digital converter (CIO-DAS1602/16, ComputerBoards).

Two sets of stimuli were devised to develop and test the models. The data obtained from the first, larger stimulus set were used to derive model parameters and provided a first test of the model's ability to predict the discriminability of the stimuli. The data from the second stimulus set were used as an additional test of the model's predictive power. The second stimulus set included triharmonic stimuli, which offered more opportunities for intercomponent interference and, thus, further challenged one of the model's central assumptions - namely, that multiple frequency components are represented quasi-independently of one another.

The first set of stimuli consisted of sinusoidal and diharmonic mechanical vibrations that varied in their frequency content and intensity. The frequencies of the simple sinusoids were 100, 150, 200, and $300 \mathrm{~Hz}$; the diharmonic stimuli were superimposed sinusoids with frequencies $100 \mathrm{~Hz}+200 \mathrm{~Hz}, 100 \mathrm{~Hz}+300 \mathrm{~Hz}$, and $150 \mathrm{~Hz}+$ $300 \mathrm{~Hz}$. The frequencies were chosen so that the stimuli vigorously stimulated the Pacinian system while eliciting little response in the RA system (Bensmaïa \& Hollins, 2000), which terminates in Meissner corpuscles at the sensory periphery. Stimulus intensities were determined with respect to the subjects' psychophysical thresholds, which were measured using a standard two-alternative forced choice paradigm in preliminary experiments. Simple (i.e., one-component) stimuli were set to three intensity levels: 17,20 , and $23 \mathrm{dBs}$ above threshold (SL) at their respective frequencies. Diharmonic stimuli were adjusted so that (1) the two frequency components of each stimulus were of equal amplitude and (2) the overall Pacinian-weighted power of each stimulus was equal to that of simple sinusoids at each of the three chosen sensation levels. Following Makous et al. (1995), the ability of each spectral component to excite the Pacinian system was expressed as a function of its spectral power $\left(A^{2} f^{2}\right)$ divided by threshold power $\left(T^{2} f^{2}\right)$ at that frequency. According to Makous et al., this quantity, summed across all frequencies, constitutes a measure of the ability of a stimulus to excite the Pacinian system. Accordingly, the amplitudes of the spectral components of each diharmonic stimulus were set using the following equation:

$$
\frac{A_{\mathrm{s}}^{2} f_{\mathrm{s}}^{2}}{T_{f_{\mathrm{s}}}^{2} f_{\mathrm{s}}^{2}}=\frac{A_{\mathrm{c}}^{2} f_{1}^{2}}{T_{f_{1}}^{2} f_{1}^{2}}+\frac{A_{\mathrm{c}}^{2} f_{2}^{2}}{T_{f_{2}}^{2} f_{2}^{2}},
$$

where $A_{\mathrm{s}}$ is the amplitude (set at 17,20 , or $23 \mathrm{db}$ SL) of a simple sinusoid at frequency $f_{\mathrm{s}}$, and $A_{\mathrm{c}}$ is the amplitude of the components of a diharmonic stimulus at frequencies $f_{1}$ and $f_{2}$. The frequency terms are left in Equation 1 for illustrative purposes but are omitted in other equations for clarity, since they systematically cancel out. The left side of the equation reduces to 50,100, or 200 for $A_{\mathrm{s}}$ 's at 17,20 , and $23 \mathrm{~dB}$ SL, respectively. Solving for $A_{\mathrm{c}}$,

$$
A_{\mathrm{c}}=\sqrt{\frac{A_{\mathrm{s}}^{2}}{T_{f_{\mathrm{s}}}^{2}} \frac{T_{f_{1}}^{2} T_{f_{2}}^{2}}{T_{f_{1}}^{2}+T_{f_{2}}^{2}} .}
$$

The first stimulus set consisted of 21 different stimuli, 12 simple and 9 diharmonic stimuli; 50-msec samples of the diharmonic waveforms are shown at the bottom right of Figure 1. 
The second stimulus set comprised complex stimuli consisting of two or three superimposed sinusoids. The frequency pairs/triplets employed were $200 \mathrm{~Hz}+400 \mathrm{~Hz}, 100 \mathrm{~Hz}+200 \mathrm{~Hz}+400 \mathrm{~Hz}, 100 \mathrm{~Hz}+$ $300 \mathrm{~Hz}+400 \mathrm{~Hz}$, and $200 \mathrm{~Hz}+300 \mathrm{~Hz}+400 \mathrm{~Hz}$. The component amplitudes for each complex waveform were again computed by a two-step process: First, the Pacinian-weighted power of the overall waveform was (as before) equated with that of a simple sinusoid at 17,20 , or $23 \mathrm{~dB}$ SL; second, the frequency components of each waveform were matched in Pacinian-weighted power, rather than (as in the first series) in amplitude. In other words, the total power of the waveform was divided by the number of spectral components, and the amplitude of each component was set so that its power was equal to that of the other component(s) in a given stimulus. We equated the components in power, rather than in amplitude, in order to ensure that the perception of polyharmonic stimuli was not dominated by one frequency component, a genuine possi-


Figure 1. Distribution of $d^{\prime}$ 's for the first data set. Top right panel shows the overall distribution; the other histograms show subsets of the data. Vertical line denotes the mean of each distribution. Bottom right panel shows 50-msec segments of the complex stimuli included in Set 1. $P_{\mathrm{S}}$ denotes Pacinian-weighted power. 
bility given the steep transfer function of the Pacinian system between 100 and $300 \mathrm{~Hz}$ (see Bensmaïa \& Hollins, 2000). The amplitude of each component was given by

$$
A_{f_{i}}=\sqrt{\frac{1}{n} \frac{A_{\mathrm{s}}^{2} T_{f_{i}}^{2}}{T_{\mathrm{s}}^{2}}},
$$

where $A_{f_{i}}$ and $T_{f_{i}}$ are the amplitude of the spectral component and threshold at frequency $f_{i}$, respectively, $n$ is the number of frequency components in the waveform (two or three), $A_{\mathrm{s}}^{2} / T_{\mathrm{s}}^{2}$ is the Pacinianweighted power of a simple sinusoid (with $A_{\mathrm{s}}$ at 17,20 , or $23 \mathrm{~dB}$ SL). The second stimulus set, then, comprised 12 different stimuli (3 with two frequency components and 9 with three); 50 -msec samples of the stimulus waveforms used in Set 2 are shown at the bottom of Figure 2.

\section{Procedure}

The subject, seated at the experimental table, rested his/her dominant hand on its surface so that the index fingertip covered the hole in the Plexiglas annulus. The contactor was then raised slowly by the experimenter $500 \mu \mathrm{m}$ beyond the point at which the subject reported barely sensing it. Earphones playing pink noise sufficiently loud to mask the sounds produced by the vibrator were then placed on the subject, and the vibrator was calibrated at all the relevant frequencies.

The calibration routine was followed by a period of vibrotactile adaptation that lasted $300 \mathrm{sec}$. Extended vibratory stimulation (adaptation) at a given frequency has been shown to reduce the sensitivity of the channel(s) that mediates the perception of that vibratory stimulus (Gescheider \& Verrillo, 1979; Gescheider \& Wright, 1968, 1969; Hollins, Goble, Whitsel, \& Tommerdahl, 1990; Verrillo \& Gescheider, 1977). The adapting stimulus was a $10-\mathrm{Hz}$ mechanical sinusoid at $30 \mathrm{~dB} \mathrm{SL}$, which was shown in a previous study (Bensmaïa \& Hollins, 2000) to raise subjects' (RA-mediated) lowfrequency thresholds by at least $10 \mathrm{~dB}$ while minimally affecting sensitivity in the high-frequency (Pacinian-mediated) range. The adapting stimulus was included in order to minimize the contribution of the RA system in the discrimination of the vibratory stimuli (see Bensmaïa \& Hollins, 2000). ${ }^{1}$

At the offset of the adapting stimulus, a 2-sec white LED switched on to signal the beginning of a discrimination trial. On each trial, the subjects were presented with a pair of waveforms (from one of the two stimulus sets) for $1 \mathrm{sec}$ each, separated by a $1-\mathrm{sec}$ inter-
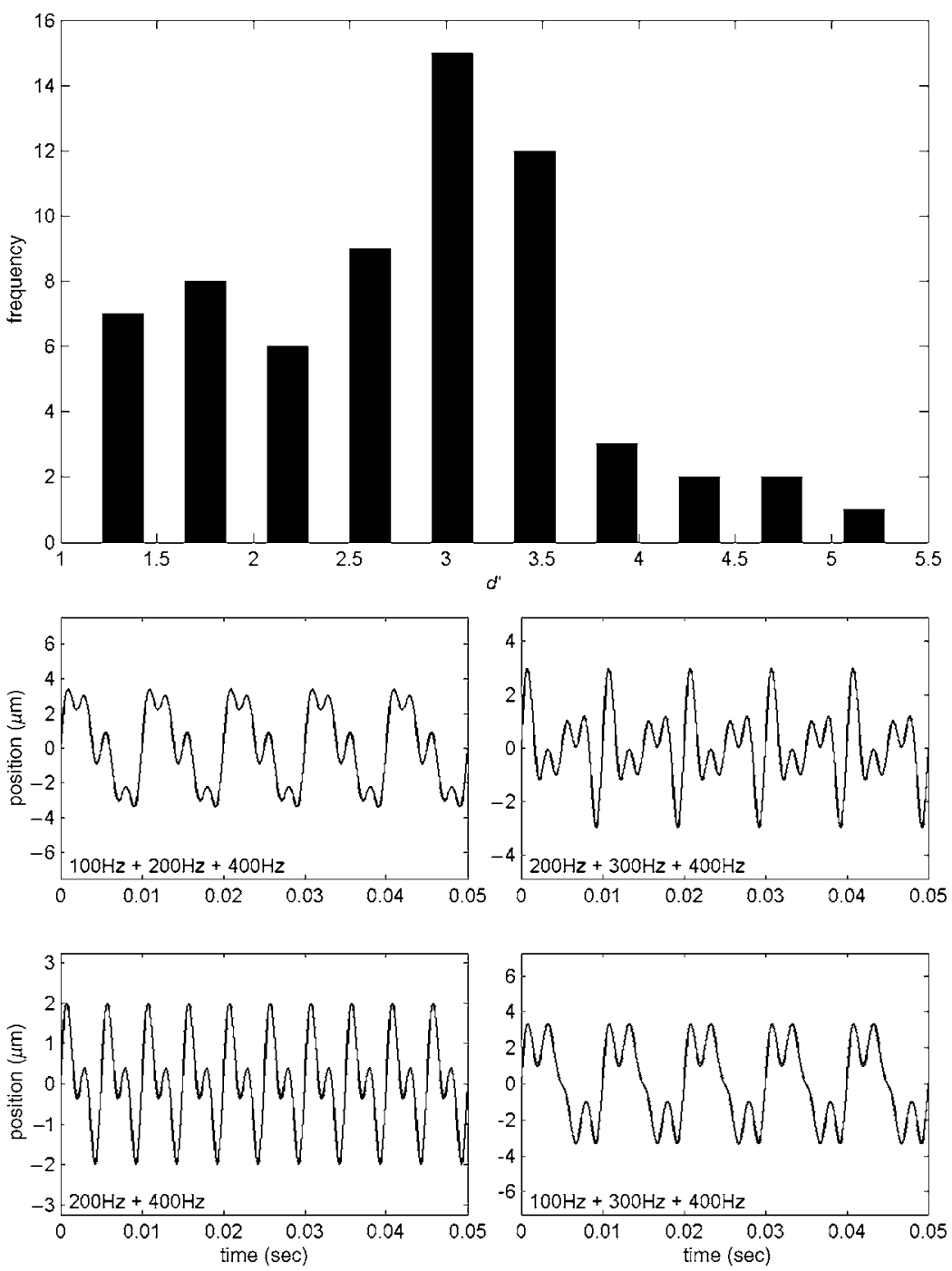

Figure 2. Distribution of $d^{\prime}$ s for the second data set. Bottom panels show 50-msec segments of the complex stimuli contained in Set 2. 
stimulus interval. The presentation of each vibrotactile stimulus was signaled by a colored LED. A fourth LED came on immediately after the offset of the second stimulus to prompt the subject for a response. The subjects were to press one button if they felt that the two waveforms had been identical and another if they felt that the two waveforms differed in any way. The subjects were told that on some trials, the two stimuli would be identical, whereas on others, they would be different. They were instructed to respond as rapidly as possible after the onset of the fourth LED. When the subject had responded, a 10-sec intertrial period began, the first $8 \mathrm{sec}$ of which contained a $10-\mathrm{Hz}, 30-\mathrm{dB}$ SL adapting stimulus to maintain the subjects' level of RA adaptation (see Bensmaïa \& Hollins, 2000). The order of presentation of the stimuli within each pair was randomized, as was that of the stimulus pairs within a run.

Each run with the first stimulus set consisted of the presentation of each of 140 pairs, including 119 different and 21 same pairs. Not all possible combinations of different pairs were presented (see Table 1). Each of six experimental sessions consisted of 2 runs; the subjects were allowed a 15-min break between runs. The first 2 runs were for practice and were not included in the analysis. Upon completion of 12 runs on the first stimulus set, the subjects followed the same procedure with the second stimulus set, which comprised 12 runs with 66 different and 24 same pairs (each same pair was presented twice to reduce bias towards different judgments). We thus obtained 10 same-different judgments from each subject for each stimulus pair from each set (except for the same pairs in the second set, for which 20 judgments were obtained).

\section{Analysis}

The raw data consisted of the proportion of trials on which each pair of stimuli was judged as different. Our objective was to derive, from these proportions, the subjects' sensitivity to differences in the frequency content and intensity of the paired stimuli. We used same-different $d$ 's, assuming a differencing rule (Sorkin, 1962), as our measure of sensitivity (cf. Bensmaïa \& Hollins, 2000). Of the two $d^{\prime}$ measures associated with the same-different paradigm, the one assuming a differencing rule is generally preferred over the one assuming independent observations (Noreen, 1981) when more than two stimuli are compared in a given run (Dai, Versfeld, \& Green, 1996; Macmillan \& Creelman, 1991). The hit rate for each different pair, then, was the proportion of times that the pair was correctly judged by each subject as different. The false alarm rate was the proportion of trials on which the subjects judged the two corresponding same pairs as different. The data for all the subjects were qualitatively similar and were, therefore, pooled. We referred to the tables in the appendix in Macmillan and Creelman (1991) to derive values of $d^{\prime}$ for each different pair.

\section{RESULTS}

Tables 1 and 2 show the discrimination data obtained from Stimulus Sets 1 and 2, respectively. Figures 1 and 2 show the distribution of $d^{\prime}$ s obtained from the two data sets. Discriminability of stimulus pairs varied over a wide range: $d^{\prime}$ ranged from 1.1 to 5.2 for Stimulus Set 1 and from 1.1 to 4.9 for Stimulus Set 2. The simple sinusoids in Data Set 1 tended to be most discriminable from one another, whereas complex stimuli were least discriminable (see Figure 1), but these differences were not great (mean $d^{\prime}$ s were 3.2 and 2.6 for simple/simple and complex/complex pairs, respectively). For both sets, the discriminability of vibratory stimuli tended to increase as the difference in their Pacinian-weighted power increased (compare panels 3, 5, and 7 in Figure 1).
However, pairs of stimuli equated for Pacinian-weighted power were often reliably discriminable; $d^{\prime}$ s for pairs of stimuli equated in intensity (using Equations 1-3) ranged from 1.2 to 4.4 for Stimulus Set 1 (see Figure 1) and from 1.3 to 3.4 for Stimulus Set 2. The critical-band hypothesis of Pacinian coding (see Makous et al., 1995) would presumably predict that high-frequency stimuli are discriminable to the extent that they differ in Pacinian-weighted power. That the stimuli equated on this property were, nonetheless, discriminable suggests that the Pacinian system conveys more than just intensive information about the stimulus.

The possibility remains that the stimuli were not equated for subjective intensity with sufficient precision. Two possible sources of error inhere in our attempts to equate subjective intensity. First, threshold estimates were liable to error, and estimates of Pacinian-weighted power may thus have been inaccurate. Error in threshold measurements would be compounded by the fact that the threshold term is squared in the expression for Pacinian-weighted power (Equations 1-3). Second, Pacinian-weighted power may be an approximation of the efficacy with which a stimulus excites the Pacinian system but may not be suited to the requirements of the same-different paradigm, in which small differences in subjective intensity may result in a sizable increment in discriminability.

\section{Intensive Model}

In order to assess the extent to which intensity cues could account for subjects' performance on the discrimination task, we implemented a general model describing the Pacinian representation of stimulus waveform exclusively in terms of spectral power. The model assumed (1) that the subjective intensity of a vibratory stimulus follows Stevens's power law (see Franzén, 1969; Marks, 1979; Stevens, 1968; Verrillo, Fraioli, \& Smith, 1969) and (2) that individual spectral components contribute independently to the overall subjective intensity of the stimulus. Note that Assumption 2 also underlies the model proposed by Makous et al. (1995). The predicted subjective intensity of Stimulus $\mathrm{S}, \Psi_{\mathrm{S}}$, was given by

$$
\Psi_{\mathrm{S}}=\sum_{i} k_{f_{i}}\left(A_{i}^{2} f_{i}^{2}\right)^{b_{f_{i}}},
$$

where $A_{i}$ and $f_{i}$ are the amplitude and frequency of spectral component $i$, respectively, and $k_{f_{i}}, b_{f_{i}}$ are free parameters that were allowed to vary from frequency to frequency. The predicted discriminability, $\Delta_{\mathrm{S}_{1} \mathrm{~S}_{2}}$, of two stimuli was proportional to the difference in their subjective intensity normalized by their average intensity (following Ekman's law2; Ekman, 1956):

$$
\Delta_{\mathrm{S}_{1} \mathrm{~S}_{2}}=\frac{\left|\Psi_{\mathrm{S}_{1}}-\Psi_{\mathrm{S}_{2}}\right|}{\frac{1}{2}\left(\Psi_{\mathrm{S}_{1}}+\Psi_{\mathrm{S}_{2}}\right)} .
$$

The model thus comprised eight free parameters: four proportionality constants $\left(k_{f_{i}}\right)$, one at each frequency, 





and four exponents $\left(b_{f_{i}}\right)$, one at each frequency. Two additional parameters - one for slope, one for interceptwere included to scale the numerical output of the model to the $d^{\prime}$ values but were not otherwise meaningful. Parameters were adjusted using the nonlinear curve-fitting algorithm of MATLAB (Version 7.0, The MathWorks).

Figure 3 shows that the intensive model was able to predict the discrimination data to a degree. The model accounted for $52 \%$ of the variance in the data obtained from Stimulus Set 1 and $60 \%$ of the variance in the data obtained from Stimulus Set 2. Furthermore, when the model was fit to data obtained from Stimulus Set 2, using the values of the parameters $k_{100 \mathrm{~Hz}}, k_{200 \mathrm{~Hz}}, k_{300 \mathrm{~Hz}}$, $b_{100 \mathrm{~Hz}}, b_{200 \mathrm{~Hz}}$, and $b_{300 \mathrm{~Hz}}$ derived using data from Stimulus Set 1 and allowing only $k_{400 \mathrm{~Hz}}$ and $b_{400 \mathrm{~Hz}}$ to vary, $56 \%$ of the variance in the data obtained from Stimulus Set 2 was accounted for by the model. Thus, not only did the intensive model account for a sizable proportion of the variance in the discrimination data, but the parameters obtained from one data set transferred to the other data set.

\section{Spectral Model}

Intensity information, then, was found to contribute to a substantial extent to the subjects' ability to discriminate high-frequency vibratory stimuli. However, a large proportion of the variance in the discrimination data remained unaccounted for by $\Delta_{\mathrm{S}_{\mathrm{S}}}$. Furthermore, the model included many degrees of freedom, which may have artificially enhanced its ability to fit the psychophysical data. We thus sought to determine whether a model that included frequency terms as arguments would be a better predictor of discriminability than one that based its prediction solely on spectral power. In order to develop such a model, we drew on extant psychophysical and neurophysiological findings. Specifically, two assumptions played a key role in determining the form of the model.

1. $\Delta f / f$ is constant across frequencies. There is disagreement as to the form of the psychometric function relating frequency difference limens (DL) to frequency of the standard. In developing the model, we assumed that the Weber fraction was constant across frequencies (see Franzén \& Nordmark, 1975; Horch, 1991; Mountcastle et al., 1990; Mountcastle et al., 1969; Rothenberg, Verrillo, Zahorian, Brachman, \& Bolanowski, 1977). The possibility that $\Delta f / f$ increases with frequency, as has been found to be the case by Goff (1967), is explored below.

2. The subjective intensity of a vibrotactile stimulus is a power function of its objective intensity. There is a general consensus in the literature that this is the case for intensities above $10 \mathrm{~dB}$ SL. The consensus exponent is around 1 with amplitude (Verrillo et al., 1969) or, equivalently, around .5 with power (Marks, 1979), although it has been found to decrease with frequency up to around 250 Hz (Franzén, 1969; Stevens, 1968).

Furthermore, we hypothesized, as we did in developing the intensive model, that the Pacinian representation of a stimulus waveform could be characterized in such a

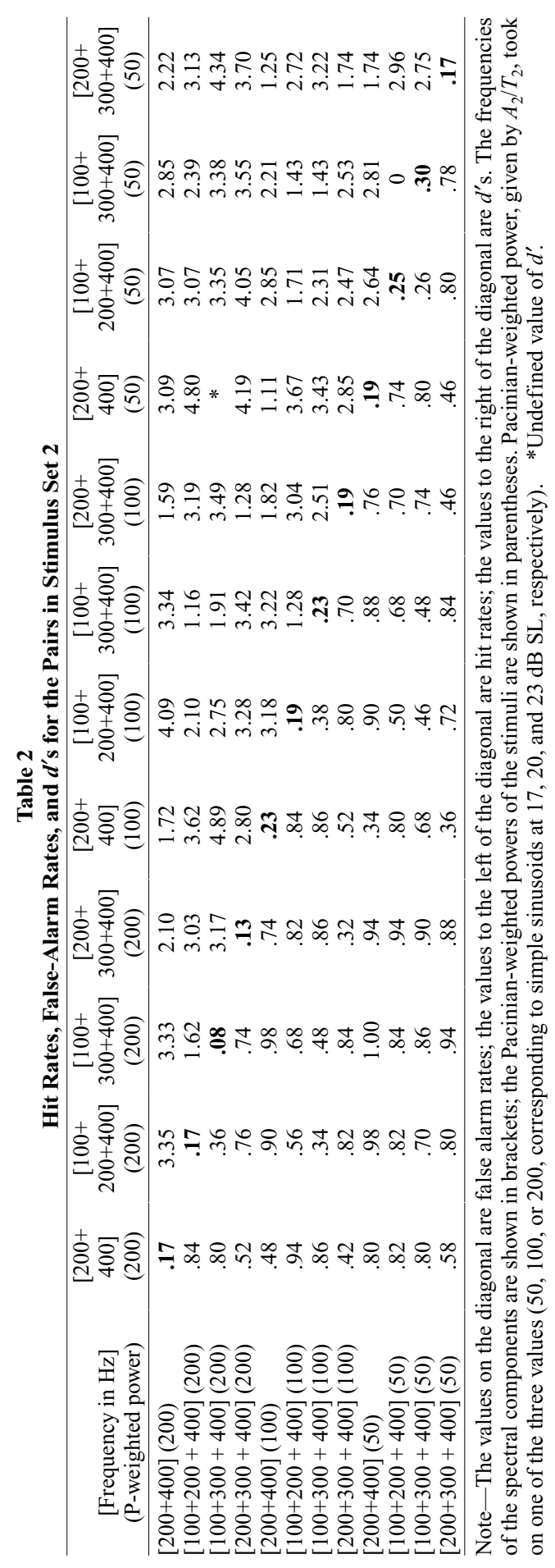





Figure 3. Discriminability $\left(d^{\prime}\right)$ vs. $\Delta_{\mathbf{S}_{1} \mathbf{S}_{2}}$, calculated from the general intensive model, is shown for the first (top) and the second (bottom) data sets. Model parameters are shown in Table 3. The general intensive model accounted for $52 \%$ of the variance in the first data set and $60 \%$ of the variance in the second data set. There were 119 data points in the first set, but only 65 in the second set (one pair was omitted since its corresponding $d^{\prime}$ was undefined; (see Table 2); the model comprised 10 free parameters.

way that each of its spectral components was represented independently of the others. This allowed us to use the power spectrum of each wave as a starting point for the analysis (see Makous et al., 1995). In addition, the activity elicited within a given minichannel by each component of a polyharmonic stimulus was additive. Our experimental results, along with results from simulations discussed below, validate this approximation.

Following the strategy used in developing the intensive model, we first devised a model characterizing the information - in this case, both intensive and temporalconveyed by the Pacinian system about each stimulus presented in the psychophysical experiment. We then derived a measure of the difference between the Pacinian representations of the two waves in each stimulus pair. We wished to assess whether this difference measure was a better predictor of the empirically determined discrim- inability $\left(d^{\prime}\right)$ than that derived from the intensive model. A good match between model predictions and data would lend support to the structure of the model and to its underlying assumptions and hypotheses.

The Pacinian representation of each stimulus, then, was modeled as a pattern of activation in a population of frequency-tuned minichannels. The overall degree of activation in the population signaled stimulus intensity, whereas the relative activation of the individual minichannels signaled frequency content.

First, the power of each component was weighted according to the frequency sensitivity of the Pacinian system (see Bensmaïa \& Hollins, 2003; Makous et al., 1995). The Pacinian-weighted power of the spectral component with frequency $f$ and amplitude $A_{f}$ in Stimulus $\mathrm{S}, P_{\mathrm{S}}(f)$, was computed as follows:

$$
P_{\mathrm{S}}(f)=\frac{A_{f}^{2}}{T_{f}^{2}},
$$

where $T_{f}$ is the threshold amplitude at $f$.

Makous et al. (1995) used $P_{\mathrm{S}}(f)$ summed across frequencies as an estimate of the ability of $\mathrm{S}$ to excite the Pacinian system. This approximation-which we invoked to set the component amplitudes of polyharmonic stimuli (see Equations 1-3) - assumes that, after accounting for threshold differences, stimuli at different frequencies stimulate the Pacinian system equally efficiently. However, exponents relating subjective to objective intensity have been found to decrease as stimulus frequency increases up to about $250 \mathrm{~Hz}$ (Franzén, 1969; Stevens, 1968), suggesting that the efficiency with which a stimulus activates the Pacinian system is a function of its frequency; taking into account changes in threshold with stimulus frequency is insufficient to capture this phenomenon.

Accordingly, the activation produced in the hypothetical minichannels by each spectral component- that is, its effective intensity - was expressed as a power function of the component's Pacinian-weighted power, the exponent of which, $a_{f}$, was a free parameter that was allowed to vary from frequency to frequency. The effective intensity $I_{\mathrm{S}}(f)$ of the frequency component at frequency $f$ in Stimulus $\mathrm{S}$ was given by

$$
I_{\mathrm{S}}(f)=\left[P_{\mathrm{S}}(f)\right]^{a_{f}} .
$$

We predicted, on the basis of the literature, that the value of $a_{f}$ would decrease with $f$ in the range of stimulus frequencies used in the present experiments.

The frequency response of each of 100 minichannels $^{3}$ was characterized by Gaussian filters with center frequency $f_{\mathrm{c}}$ and standard deviation $\alpha \cdot f_{\mathrm{c}}$ (center frequencies were spaced in equal logarithmic increments between 1 and $1000 \mathrm{~Hz}$, see the top of Figure 4). Gaussian filters were used to describe frequency tuning, since they comprise few parameters and are symmetric about a maximum, thus lending themselves well to modeling this phenomenon. The increase in filter width with $f_{\mathrm{c}}$ consti- 


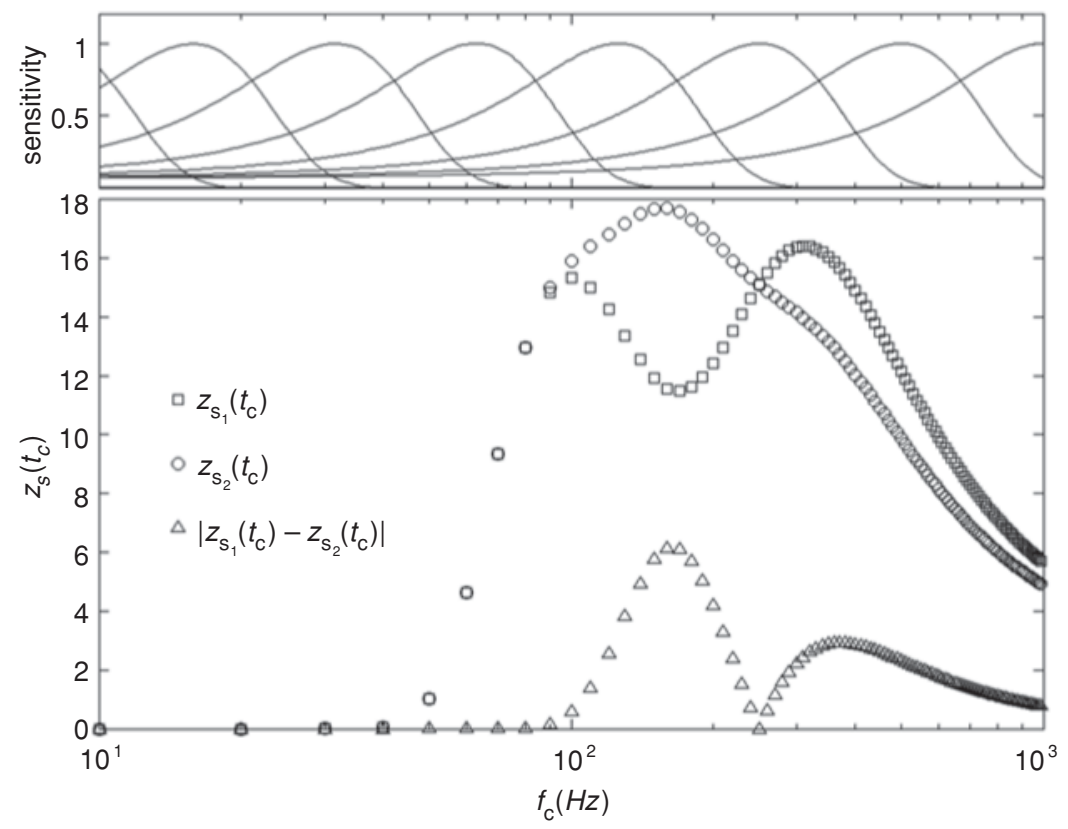

Figure 4. Examples of patterns of activation in the hypothetical population of frequency-tuned minichannels, according to the spectral model. The patterns shown by the squares and circles, respectively, were elicited by triharmonic stimuli $\left(S_{1}\right.$ and $S_{2}$ ) from Set 2: $S_{1}$ consists of superimposed sinusoids at 100,200 , and $400 \mathrm{~Hz} ; S_{2}$ consists of superimposed sinusoids at 100,300 , and $400 \mathrm{~Hz}$. The absolute differences between the two activation levels of each minichannel are represented by the triangles. The inset at the top shows the spectral sensitivity profiles of a subset of minichannels.

tuted an implementation of the assumption that $\Delta f / f$ is constant across $f$ (see below). The activation elicited in each minichannel by multiple frequency components was additive:

$$
Z_{\mathrm{S}}\left(f_{\mathrm{c}}\right)=\sum_{f} I_{\mathrm{S}}(f) \cdot e^{-\frac{\left(f-f_{\mathrm{c}}\right)^{2}}{2\left(\alpha f_{\mathrm{c}}\right)^{2}}},
$$

where $Z_{\mathrm{S}}\left(f_{\mathrm{c}}\right)$ denotes the activation of the minichannel with central frequency $f_{\mathrm{c}}$ by Stimulus $\mathrm{S}$.

In order to assess how well our model captured the informational content of the Pacinian-mediated representation of simple and complex waveforms, we evaluated the extent to which differences in $Z_{\mathrm{S}}\left(f_{\mathrm{c}}\right)$ summed across $f_{\mathrm{c}}$ could predict our measured $d^{\prime}$ s. To that end, we used the absolute difference in $Z_{\mathrm{S}}\left(f_{\mathrm{c}}\right)$ across central frequencies, normalized following Ekman's (1956) law by the mean activity elicited by the two stimuli, as a measure of the spectral dissimilarity, $D_{\mathrm{S}_{1} \mathrm{~S}_{2}}$, of Stimuli $\mathrm{S}_{1}$ and $\mathrm{S}_{2}$ :

$$
D_{\mathrm{S}_{1} \mathrm{~S}_{2}}=\frac{\sum_{f_{\mathrm{c}}}\left|Z_{\mathrm{S}_{1}}\left(f_{\mathrm{c}}\right)-Z_{\mathrm{S}_{2}}\left(f_{\mathrm{c}}\right)\right|}{\frac{1}{2}\left(\sum_{f_{\mathrm{c}}} Z_{\mathrm{S}_{1}}\left(f_{\mathrm{c}}\right)+\sum_{f_{\mathrm{c}}} Z_{\mathrm{S}_{2}}\left(f_{\mathrm{c}}\right)\right)} .
$$

$D_{\mathrm{S}_{1} \mathrm{~S}_{2}}$ was optimized by searching for values of the parameters $\left[\alpha, a_{f_{1}}, a_{f_{2}}, a_{f_{3}}, a_{f_{4}}\right]$ that yielded the highest correlation between $D_{\mathrm{S}_{1} \mathrm{~S}_{2}}$ and the $d^{\prime}$ 's obtained from the first stimulus set. Since the frequencies used in the first set were $100,150,200$, and $300 \mathrm{~Hz}$, no exponent was obtained at $400 \mathrm{~Hz}$ from the initial fit. Since $400 \mathrm{~Hz}$ was one of the frequencies used in the second stimulus set, the exponent $a_{400 \mathrm{~Hz}}$ was the only parameter allowed to vary to fit the model to these data, with the other parameters set to the values derived from the first data set. Figure 4 shows the patterns of activation, $Z_{\mathrm{S}}\left(f_{\mathrm{c}}\right)$, elicited in the hypothetical population of minichannels by two triharmonic stimuli differing in their frequency content (but equated for Pacinian-weighted power) as a function of $f_{\mathrm{c}}$; also shown is the absolute difference between the two patterns.

The model allowed us to estimate (1) the uncertainty in the temporal information conveyed by the Pacinian system as indexed by $\alpha$ (and thus, the Weber fraction for frequency DLs) and (2) the exponents relating effective intensity to Pacinian-weighted power at 100, 150, 200, 300 , and $400 \mathrm{~Hz}$. We could then compare the optimal values of these parameters with analogous measures obtained in other experimental contexts.

When the model was fit to the data obtained from the first stimulus set, the best-fitting values for parameters $\left[\alpha, a_{100 \mathrm{~Hz}}, a_{150 \mathrm{~Hz}}, a_{200 \mathrm{~Hz}}, a_{300 \mathrm{~Hz}}\right]$ were $[0.43,0.65,0.60$, $0.51,0.52]$ yielding a correlation between $D_{\mathrm{S}_{1} \mathrm{~S}_{2}}$ and $d^{\prime}$ of $\rho=.89$. Figure 5 shows the extent to which the model fit is affected by changes in the parameter values; note that the plots are rather sharply peaked at the optimal values. Furthermore, at high values of $\alpha$, the model re- 

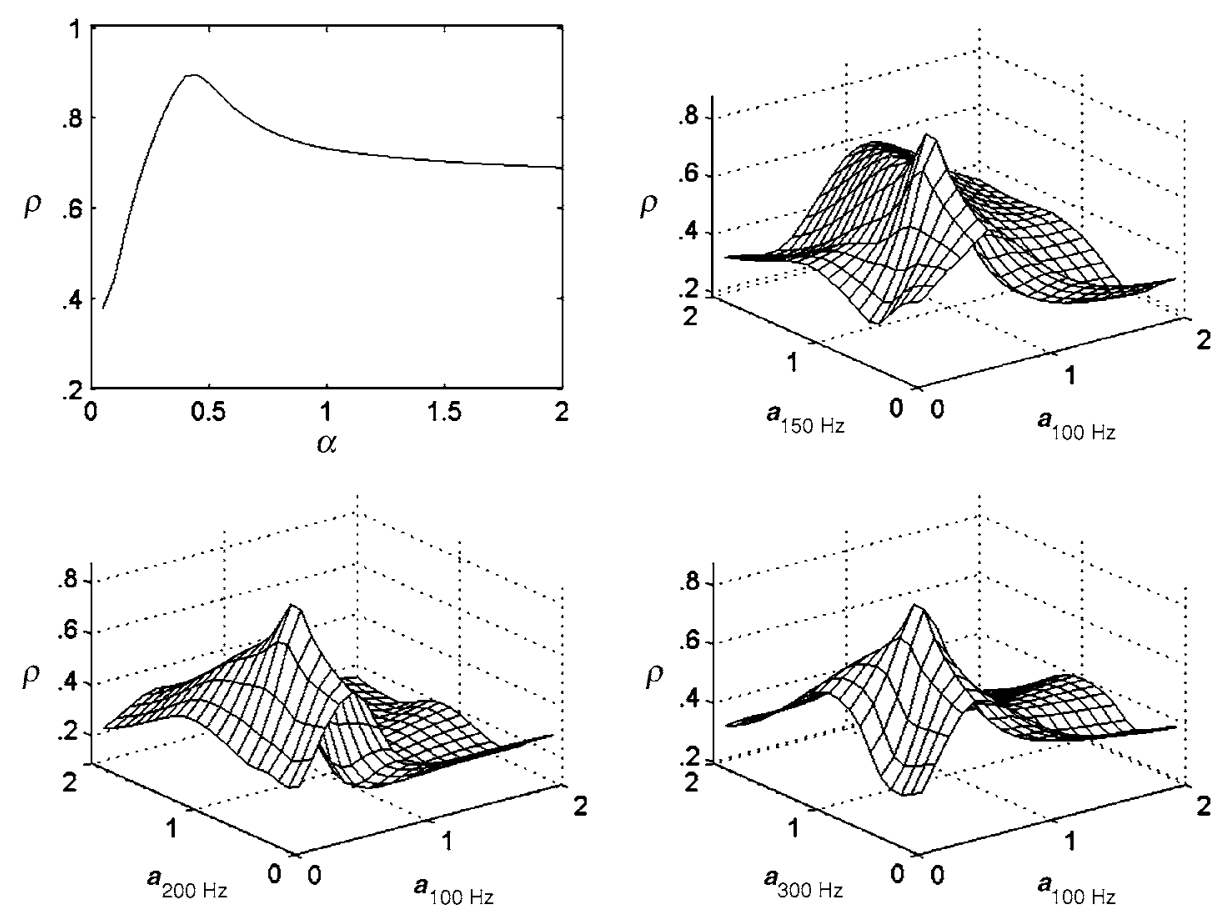

Figure 5. Model fit as a function of model parameters. The top left panel shows the correlation between model and data as a function of $\alpha$ when the exponents are set to their optimal values. The other three panels show the effects of varying $a_{100 \mathrm{~Hz}}$ and one of the other three parameters on model fit, with the other parameters set to their optimal values.

duces to an intensive model, since the minichannels exhibit uniform frequency selectivity.

When the model was fit to the data obtained from the second stimulus set by varying only $a_{400 \mathrm{~Hz}}$ (using the values for $\left[\alpha, a_{100 \mathrm{~Hz}}, a_{200 \mathrm{~Hz}}, a_{300 \mathrm{~Hz}}\right]$ obtained from the first set), the best-fitting value of the free parameter was found to be 0.35 , yielding a correlation between $D_{\mathrm{S}_{1} \mathrm{~S}_{2}}$ and $d^{\prime}$ of $\rho=.88$. A plot of $d^{\prime}$ versus $D_{\mathrm{S}_{1} \mathrm{~S}_{2}}$ for each data set is shown in Figure 6.

In order to derive a statistical measure of fit, we used a Monte Carlo approach to generate a sampling distribution for $\rho$ when the model was fit to $d^{\prime}$ 's sampled without replacement from the set of all $d^{\prime}$ s. For each set of sampled $d$ 's, we searched for values of the five parameters that yielded the highest $\rho$. We sampled 500 optimized correlation coefficients, using $d$ 's from each data set, yielding two sampling distributions, which we found to be approximately normal. We then estimated that the probability of obtaining, by chance, a correlation coefficient of .89 was $7.7 \times 10^{-26}$, given the model and the stimuli in Set 1, and that of obtaining a correlation of .88 was $7.1 \times 10^{-13}$, given the model and the stimuli in Set $2 .{ }^{4}$ In other words, the high degree of fit between model predictions and data is very unlikely to be the result of chance. Furthermore, the variance in the discriminability data accounted for by the spectral model was significantly greater than that accounted for by the intensive model $\left(\Delta R^{2}=.27\right.$ and $.17, F=178.3$ and 67.7 for Stimulus Sets 1 and 2 , respectively, $p<.001$ ).

\section{Discussion}

The major finding of this study is that precise temporal information about high-frequency vibrotactile stimuli, which has been known since the work of Talbot et al. (1968) to be carried by Pacinian afferents, plays a role in perception. In other words, we have shown that discrimination of high-frequency vibrotactile stimuli, the perception of which is mediated by the Pacinian system, is too rich and precise to be based on intensive information alone.

There has been some earlier evidence of utilization of temporal cues at high frequencies (Franzén \& Nordmark, 1975), but other work has seemed to indicate the opposite. Specifically, Goff's (1967) finding that the Weber fraction of vibrotactile frequency discrimination increases with the frequency of the standard seems to suggest that frequency discrimination might require the participation of non-Pacinian channels, and Makous et al.'s (1995) discovery that, with regard to masking, the Pacinian channel acts like a critical band gives no hint of frequency-specific effects. However, Goff's results may reflect an incomplete following of the vibrotactile waveform by the skin, given the low pressure of the contactor in her study; and the fact that the Pacinian channel masks as a unit (Makous et al., 1995) does not mean that temporal information is not used for other purposes.

The present study shows that intensive cues contribute importantly to the discriminability of high-frequency stimuli (both simple and complex) but that such cues by 

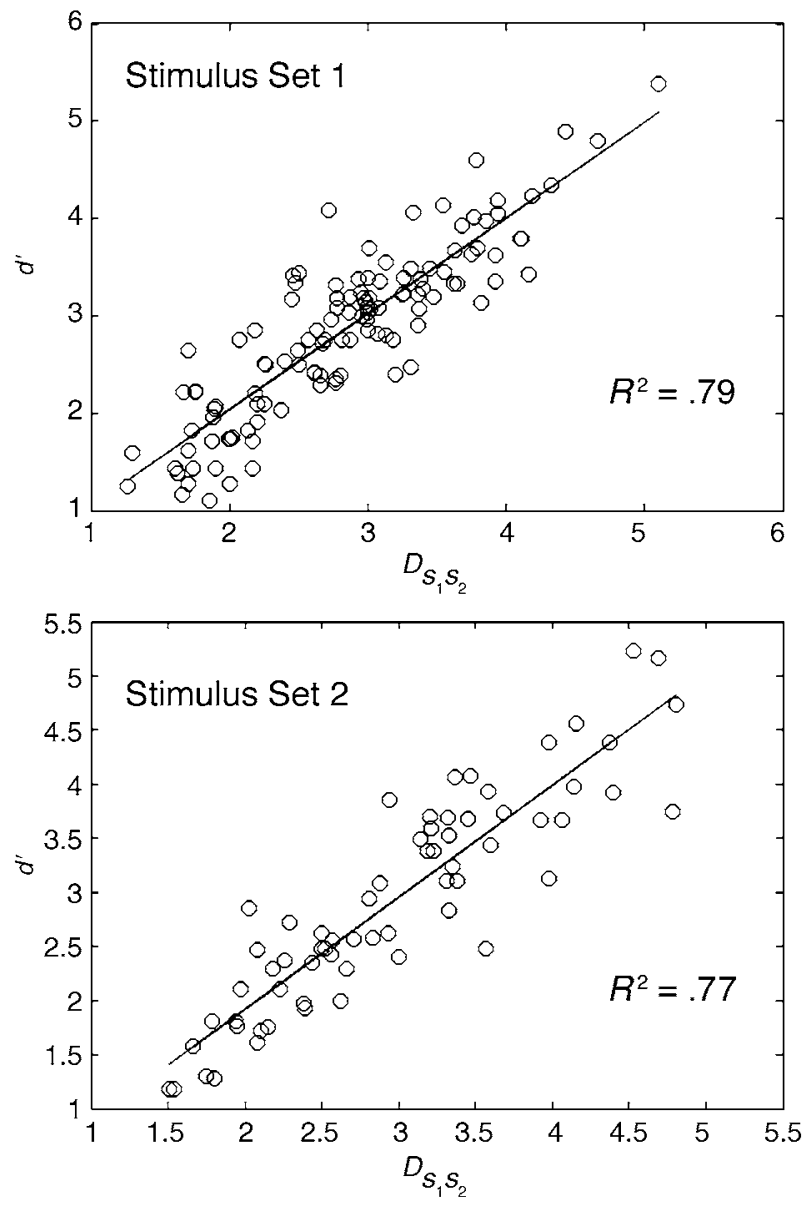

Figure 6. $d^{\prime}$ versus $D_{\mathrm{S}_{1} \mathrm{~S}_{2}}$ obtained from the first (top) and second (bottom) stimulus sets with $\alpha=0.43, a_{100 \mathrm{~Hz}}=0.65, a_{150 \mathrm{~Hz}}=$ $0.60, a_{200 \mathrm{~Hz}}=0.51, a_{300 \mathrm{~Hz}}=0.52$, and $a_{400 \mathrm{~Hz}}=0.34$. The probabilities of obtaining correlation coefficients of .89 and .88 , given scrambled sets, are $7.7 \times 10^{-26}$ and $7.1 \times 10^{-13}$ for Stimulus Sets 1 and 2 , respectively.

themselves are not sufficient. Temporal information must also be taken into account to explain the discrimination performance that we have observed. It is not simply the keenness of discrimination that is probative, but the patterns of discrimination across pairs of stimuli, for these patterns are well captured by a psychophysical model that combines, in a novel but plausible way, established principles, such as Ekman's law and Stevens' law, with the idea of frequency-specific processing modules (minichannels) that collectively capture temporal information.

\section{Model Parameters}

An important test of the model is whether its parameters have values that are reasonable, given what has been learned about the Pacinian system from earlier studies. Consider, for example, the width of the Gaussian filters characterizing the frequency tuning of the minichannels, which is specified by the parameter $\alpha$. This parameter is a measure of the uncertainty in the fre- quency information conveyed by the Pacinian system: As $\alpha$ increases, frequency information becomes less defined, because the amount of overlap in the minichannels' frequency tuning increases. Because $\alpha$ is a multiplicative factor, the model assumes that temporal uncertainty increases linearly with stimulus frequency. In fact, the model predicts that the Weber fraction for frequency discrimination remains constant across frequencies. To estimate the Weber fraction predicted by the model, we presented the model (with $\alpha$ fixed at 0.43 ) with pairs of simple sinusoids, which were initially set to the same frequency. The frequency of the second sinusoid was incremented until $D_{\mathrm{S}_{\mathrm{S}} \mathrm{S}_{2}}$ assumed a value of 1.35 (which corresponds to a traditional threshold $d^{\prime}$ ). This process was repeated using different standard frequencies. The Weber fraction predicted by the model was found to be constant at 0.22 and matched almost exactly that measured by Mountcastle et al. (1969) and Rothenberg et al. (1977) for sinusoidal stimuli.

The optimal values of the exponents characterizing the efficiency of components at different frequencies to activate the Pacinian system are also compatible with measured values of exponents relating subjective to objective intensity. For frequencies between 100 and $400 \mathrm{~Hz}$, measured exponents range from 0.29 to 0.5 with power as the intensity measure (Franzén, 1969; Stevens, 1968; Verrillo et al., 1969), whereas the values derived from the model range from 0.35 to 0.65 . Furthermore, the derived exponents tend to decrease with frequency from a high of 0.65 at $100 \mathrm{~Hz}$ to a low of 0.35 at $400 \mathrm{~Hz}$, paralleling the decrease in exponents reported by Stevens (1968) and Franzén (1969) for psychophysical magnitude functions measured with sinusoids on the fingertip. When the model parameters were set to values interpolated from those measured by Franzén, ${ }^{5}$ the correlations between $D_{\mathrm{S}_{1} \mathrm{~S}_{2}}$ and $d^{\prime}$ were .75 and .76 for Stimulus Sets 1 and 2 , respectively, further indicative of the match between the derived exponents and exponents measured in other psychophysical contexts.

Table 3

Intensive Model Parameters

\begin{tabular}{cccc}
\multicolumn{4}{c}{ Intensive } \\
\hline & Set 1 & Set 2 & Set 2 (1) \\
\hline$k_{100 \mathrm{~Hz}}$ & 1.52 & -0.15 & 1.52 \\
$k_{150 \mathrm{~Hz}}$ & 0.77 & - & - \\
$k_{200 \mathrm{~Hz}}$ & 0.11 & 3.74 & 0.11 \\
$k_{300 \mathrm{~Hz}}$ & 2.78 & 0.56 & 2.78 \\
$k_{400 \mathrm{~Hz}}$ & - & 0.00 & 0.07 \\
$b_{100 \mathrm{~Hz}}$ & 0.92 & 1.08 & 0.92 \\
$b_{150 \mathrm{~Hz}}$ & 0.77 & - & - \\
$b_{200 \mathrm{~Hz}}$ & 0.96 & 0.98 & 0.96 \\
$b_{300 \mathrm{~Hz}}$ & 0.17 & 1.03 & 0.17 \\
$b_{400 \mathrm{~Hz}}$ & - & -1.19 & 0.95 \\
\hline
\end{tabular}

Note-The first set of parameters was obtained when the model was fitted to the data from Stimulus Set 1; the second set of parameters was obtained when the model was fitted to the data from Stimulus Set 2. The third set of parameters was obtained by setting $k_{100 \mathrm{~Hz}}, k_{200 \mathrm{~Hz}}, k_{300 \mathrm{~Hz}}$, $b_{100 \mathrm{~Hz}}, b_{200 \mathrm{~Hz}}$, and $b_{300 \mathrm{~Hz}}$ to values obtained from the first stimulus set and allowing $k_{400 \mathrm{~Hz}}$ and $b_{400 \mathrm{~Hz}}$ to vary. 


\section{Model Assumptions}

One of the central assumptions underlying the model is that $\Delta f / f$ is constant across frequencies (Franzén \& Nordmark, 1975; Horch, 1991; Mountcastle et al., 1969; Rothenberg et al., 1977). The only other plausible assumption would be that Weber fractions increase with frequency, as has been found to be the case by Goff (1967). We tested this possibility by implementing a model that produces Weber fractions that increase linearly with frequency (by setting the standard deviation of the Gaussians to $\alpha f^{2}$ ). This alternate model, which was otherwise identical to the original, was once again optimized by searching for values of $\left[\alpha, a_{f_{1}}, a_{f_{2}}, a_{f_{3}}, a_{f_{4}}\right]$ that yielded the highest correlation between $D_{\mathrm{S}_{1} \mathrm{~S}_{2}}$ and $d^{\prime}$. The fit achieved using this alternate model was inferior to that obtained with the original $(\rho=.80$ and .65 for Stimulus Sets 1 and 2, respectively), which lends support to the hypothesis that the Weber fraction for frequency DLs is constant across frequencies. ${ }^{6}$

Another significant assumption underlying the proposed model is that each frequency component is represented approximately independently of others since (1) the same model and parameter values are used to predict the discriminability of simple and complex stimuli and (2) the activity elicited in the minichannels by multiple components is additive (Equation 8). Two results of the modeling effort support this assumption. First, the fit between model and data is equivalent for pairs of simple sinusoids, pairs containing at least one diharmonic stimulus, and pairs containing two polyharmonic stimuli ( $\rho=.90, .88$, and .87 , respectively). Thus, the same set of parameters applies about equally well to simple sine waves and polyharmonic stimuli. Second, the optimal values of the parameters are comparable to values measured using simple sinusoids as stimuli. The value of the Weber fraction for frequency discrimination predicted by the model, 0.22 , was compatible with previously measured values. The optimal exponents are also similar to analogous values obtained in previous studies. If the representations of individual components interfered with one another, one might predict that the Weber fraction predicted on the basis of experiments with complex waveforms would be higher and the predicted exponents lower than values derived from experiments with simple sinusoids; component frequencies and amplitudes would be less discriminable in polyharmonic than in simple stimuli.

The question remains, then, how Pacinian afferents convey information about multiple frequency components simultaneously. There is no evidence to suggest that there are systematic differences in the frequency response curves of Pacinian corpuscles (Bolanowski \& Zwislocki, 1984; Talbot et al., 1968) such that stimuli at different frequencies might engage different (albeit overlapping) populations of receptors (as is the case, for example, in the cochlea). Another possibility, however, is that Pacinian corpuscles that are differentially sensitive to the stimulus, due either to intrinsic differences in sensitivity or to differences in their locations with respect to the locus of stimulation, might become entrained with different frequency components. In other words, a receptor near the vibrator may respond to one subset of frequency components, whereas one further away may respond to another.

To test this hypothesis, we simulated a set of Pacinian afferents and examined the frequency content of their responses to a triharmonic stimulus consisting of superimposed sinusoids at 100, 200, and $500 \mathrm{~Hz}$. Individual afferents were simulated using a simple integrate-andfire receptor model similar to that described by Bensmaïa (2002) for the Meissner corpuscle, itself inspired by a model developed by Freeman and Johnson (1982a, 1982b; Slavík \& Bell, 1995). Briefly, the Pacinian afferent model consisted of two components: a transduction site and a spike initiation site. Stimulus acceleration was integrated at the transduction site, modeled as a leaky membrane. When the receptor potential reached a critical level, an action potential was produced at the spike initiation site, and the receptor potential was reset to 0 . Acceleration was used as the critical stimulus quantity, on the basis of the finding that, when responding to nonrepeating noise sequences, Pacinian afferents tend to fire when the stimulus acceleration is high (Looft, 1996).

Each afferent's response, then, was characterized as follows.

1 . The receptor potential, initialized at 0 , becomes depolarized as a function of stimulus acceleration, so that

$$
P_{t}=P_{t-1}+\gamma a_{t}-P_{t} / \tau,
$$

where $P_{t}$ and $a_{t}$ are the membrane potential and stimulus acceleration at time $t$, respectively, $\gamma$ is the gain, and $\tau$ is the membrane time constant (set to $3.4 \mathrm{msec}$; see Freeman \& Johnson, 1982a). Receptors half-wave rectify the stimuli so that they are sensitive only to positive values of $a_{t}$ (if $a_{t}<0, a_{t} \rightarrow 0$; see Bensmaïa, 2002). The time increment was $10 \mu \mathrm{sec}$.

2 . If $P_{t}$ reaches or exceeds threshold (set at 1), the afferent produces an action potential, the time $t$ is recorded, $P_{t}$ is reset to 0 , and the receptor becomes absolutely refractory for $1 \mathrm{msec}$ (Bensmaïa, 2002; Freeman \& Johnson, 1982b; Slavík \& Bell, 1995).

We simulated the responses of a population of afferents that differed systematically in their sensitivity to the stimulus (designated by the parameter $\gamma$ ). Again, differences in $\gamma$ represent either intrinsic differences in receptor sensitivity or differences in receptor position, relative to the locus of stimulation. We found that different afferents became entrained with different stimulus components or combinations of components (see Figure 7). The least sensitive afferents tended to be entrained exclusively with low-frequency components and afferents of intermediate sensitivity responded, instead, to intermediate- and highfrequency components, whereas the most sensitive afferents responded to combinations of frequency components.

The significant result, then, is that different frequency components can be represented simultaneously in a population of afferents having identical spectral profiles. 


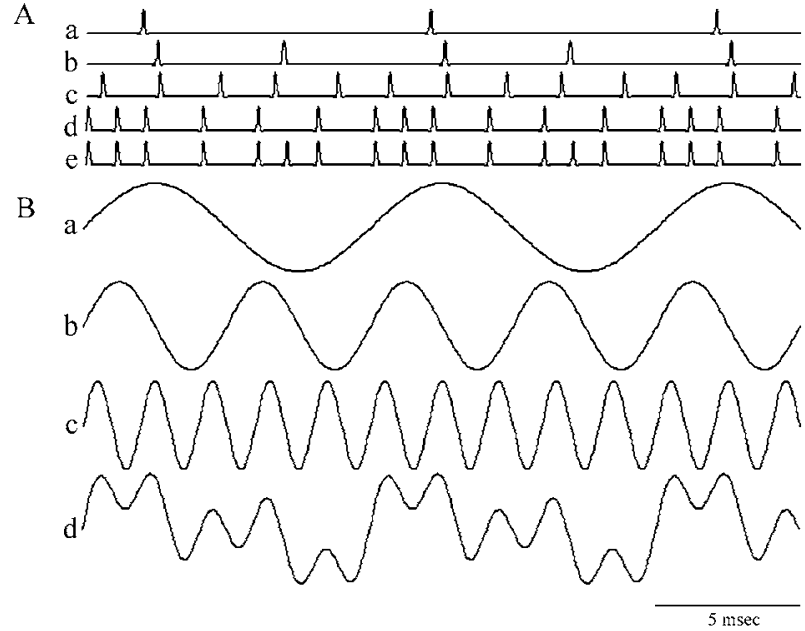

Figure 7. Response of a subset of simulated receptors to three cycles of a complex waveform consisting of three frequency components at 100, 200, and $500 \mathrm{~Hz}$. (A) Raster plot representing the pattern of firing for a simulated corpuscle that fires (a) on every cycle of the $100-\mathrm{Hz}$ component, (b) on every cycle of the $200-\mathrm{Hz}$ component, (c) on every cycle of the $500-\mathrm{Hz}$ component, (d) on every cycle of the 100- and 500-Hz components, and (e) on every cycle of the 200- and 500-Hz components. (B) (a) 100-Hz component; (b) 200-Hz component; (c) 500-Hz component; (d) the triharmonic stimulus comprising frequency components at $\mathbf{1 0 0}$, 200 , and $500 \mathrm{~Hz}$ at equal amplitudes.

The particulars of the afferent model played little role in obtaining this result. For instance, whether Pacinian corpuscles are sensitive to acceleration or jerk (see Johnson, 2001) changes only the relative sensitivity of the receptor to the various frequency components of the stimulus (amplitude is weighted by $f^{2}$ for acceleration and by $f^{3}$ for jerk). A model that assumes jerk to be the relevant stimulus parameter would yield a greater preponderance of fibers firing at the high frequencies than would one based on acceleration. Other aspects of the model, such as the specific value of the time constant or the duration (or indeed, inclusion) of the refractory period, are equally irrelevant.

In summary, a model that characterizes the Pacinian representation of an arbitrary vibratory stimulus as a pattern of activity in a population of frequency-tuned minichannels has been shown to be an excellent predictor of the discriminability of high-frequency stimuli. The model does not capture every known property of the vibratory sense. For instance, frequency DLs have been shown to decrease as stimulus amplitude increases (Goff, 1967; LaMotte \& Mountcastle, 1975), a phenomenon not predicted by the model. However, the model can be easily modified to exhibit this characteristic (for instance, by including a term in the standard deviation of the Gaussians that causes the tuning curves to become narrower as stimulus intensity increases). Furthermore, we expect that the representation of certain stimuli, such as stimuli containing beats, will not be accurately captured by the present model (see Morley et al., 1990). However, we hope that the present study will constitute a foundation leading toward a better understanding of how the Pacinian system - and more broadly, the vibrotactile system - simultaneously conveys temporal and intensive information.

\section{REFERENCES}

BensmaÏA, S. J. (2002). A transduction model of the Meissner corpuscle. Mathematical Biosciences, 176, 203-217.

BensmaÏA, S. J., \& Hollins, M. (2000). Complex tactile waveform discrimination. Journal of the Acoustical Society of America, 108, 12361245.

Bensmaïa, S. J., \& Hollins, M. (2003). The vibrations of texture. Somatosensory \& Motor Research, 20, 33-43.

Bolanowski, S. J., Jr., Gescheider, G. A., Verrillo, R. T., \& CheckOSKY, C. M. (1988). Four channels mediate the mechanical aspects of touch. Journal of the Acoustical Society of America, 84, 1680-1694.

BolanOWSKI, S. J., JR., \& ZWISLOCKI, J. J. (1984). Intensity and frequency characteristics of Pacinian corpuscles: I. Action potentials. Journal of Neurophysiology, 51, 793-811.

DaI, H., Versfeld, N. J., \& Green, D. M. (1996). The optimum decision rules in the same-different paradigm. Perception \& Psychophysics, 58, 1-9.

DeValois, R. L., \& DeValois, K. K. (1988). Spatial vision. Oxford: Oxford University Press.

EKMAN, G. (1956). Discriminal sensitivity on the subjective continuum. Acta Psychologica, 12, 233-243.

Ferrington, D. G., \& Rowe, M. (1980). Differential contributions to coding of cutaneous vibratory information by cortical somatosensory areas I and II. Journal of Neurophysiology, 43, 310-331.

Formby, C., Morgan, L. N., Forrest, T. G., \& Raney, J. J. (1992). The role of frequency selectivity in measures of auditory vibrotactile resolution. Journal of the Acoustical Society of America, 91, 293-305.

FrAnZÉN, O. (1969). The dependence of vibrotactile threshold and magnitude functions on stimulation frequency and signal level: A perceptual and neural comparison. Scandinavian Journal of Psychology, 10, 289-298.

FranzÉN, O., \& NordMARK, J. (1975). Vibrotactile frequency discrimination. Perception \& Psychophysics, 17, 480-484.

Freeman, A. W., \& Johnson, K. O. (1982a). Cutaneous mechanoreceptors in macaque monkey: Temporal discharge patterns evoked by vibration, and a receptor model. Journal of Physiology, 323, 21-41.

Freeman A. W., \& Johnson, K. O. (1982b). A model accounting for effects of vibratory amplitude on responses of cutaneous mechanoreceptors in macaque monkey. Journal of Neurophysiology, 323, 43-64.

Gescheider, G. A., \& VerriLlo, R. T. (1979). Vibrotactile frequency characteristics as determined by adaptation and masking procedures. In D. Kenshalo (Ed.), Sensory functions of the skin of humans (pp. 183-206). New York: Plenum.

Gescheider, G. A., \& Wright, J. H. (1968). Effects of sensory adaptation on the form of the psychophysical magnitude function for cutaneous vibration. Journal of Experimental Psychology, 77, 308-313.

Gescheider, G. A., \& Wright, J. H. (1969). Effects of vibrotactile adaptation on the perception of stimuli of varied intensity. Journal of Experimental Psychology, 80, 449-453.

GoFf, G. D. (1967). Differential discrimination of frequency of cutaneous mechanical vibration. Journal of Experimental Psychology, 74, 294-299.

Hollins, M., Goble, A. K., Whitsel, B. L., \& Tommerdahl, M. (1990). Time course and action spectrum of vibrotactile adaptation. Somatosensory \& Motor Research, 7, 205-221.

Horch, K. (1991). Coding of vibrotactile stimulus frequency by Pacinian corpuscle afferents. Journal of the Acoustical Society of America, 89, 2827-2836.

Hyvärinen, J., Sakata, H., Talbot, W. H., \& Mountcastle, V. B. (1968). Neuronal coding by cortical cells of frequency of oscillating peripheral stimuli. Science, 162, 1130-1132.

JoHnson, K. O. (2001). The roles and functions of cutaneous mechanoreceptors. Current Opinions in Neurobiology, 11, 455-461.

Lamoré, P. J. J., MuiJser, H., \& Keemink, C. J. (1986). Envelope detection of amplitude-modulated high-frequency sinusoidal signals by 
skin mechanoreceptors. Journal of the Acoustical Society of America, 79, 1082-1085.

LaMotte, R. H., \& Mountcastle, V. B. (1975). Capacities of humans and monkeys to discriminate vibratory stimuli of different frequency and amplitude: A correlation between neural events and psychological measurements. Journal of Neurophysiology, 38, 539-559.

LoofT, F. J. (1996). Responses of monkey glabrous skin mechanoreceptors to random noise sequences: II. Dynamic stimulus state analysis. Somatosensory \& Motor Research, 13, 11-28.

Macmillan, N. A., \& Creelman, C. D. (1991). Detection theory: A user's guide. Cambridge: Cambridge University Press.

Makous, J. C., Friedman, R. M., \& Vierck, C. J., JR. (1995). A critical band filter in touch. Journal of Neuroscience, 15, 2808-2818.

MarKs, L. E. (1979). Summation of vibrotactile intensity: An analog to auditory critical bands? Sensory Processes, 3, 188-203.

Moore, B. C., \& Patterson, R. D. (1986). Auditory frequency selectivity. New York: Plenum.

Morley, J. W., Archer, J. S., Ferrington, D. G., Rowe, M. J., \& TurMAN, A. B. (1990). Neural coding of complex tactile vibration. In M. Rowe \& L. Aitkin (Eds.), Information processing in mammalian auditory and tactile systems (pp. 127-140). New York: Wiley-Liss.

Mountcastle, V. B., Steinmetz, M. A., \& Romo, R. (1990). Frequency discrimination in the sense of flutter: Psychophysical measurements correlated with postcentral events in behaving monkeys. Journal of Neuroscience, 10, 3032-3044.

Mountcastle, V. B., Talbot, W. H., Darian-Smith I., \& Kornhuber, H. H. (1967). Neural basis of the sense of flutter-vibration. Science, 155, 597-600.

Mountcastle, V. B., Talbot, W. H., Sakata, H., \& Hyvärinen, J. (1969). Cortical neuronal mechanisms in flutter-vibration studied in unanesthetized monkeys: Neuronal periodicity and frequency discrimination. Journal of Neurophysiology, 32, 452-483.

NoreEn, D. L. (1981). Optimal decision rules for common psychophysical paradigms. In S. Grossberg (Ed.), Mathematical psychology and psychophysiology (pp. 237-280). Hillsdale, NJ: Erlbaum.

OCHOA, J., \& TOREBJöRK, E. (1983). Sensations evoked by intraneural microstimulation of single mechanoreceptor units innervating the human hand. Journal of Physiology, 342, 633-654.

Rothenberg, M., Verrillo, R. T., Zahorian, S. A., Brachman, M. L., \& BolanowsKI, S. J., JR. (1977). Vibrotactile frequency for encoding a speech parameter. Journal of the Acoustical Society of America, 62, 1003-1012.

Slavík, P., \& Bell, J. (1995). A mechanoreceptor model for rapidly and slowly adapting afferents subjected to periodic vibratory stimuli. Mathematical Biosciences, 130, 1-23.

SORKIN, R. D. (1962). Extensions of the theory of signal detectability to matching procedures in psychoacoustics. Journal of the Acoustical Society of America, 34, 1745-1751.

Stevens, S. S. (1968). Tactile vibration: Change of exponent with frequency. Perception \& Psychophysics, 3, 223-228.

Talbot, W. H., Darian-Smith, I., Kornhuber, H. H., \& MountcasTLE, V. B. (1968). The sense of flutter-vibration: Comparison of the human capacity with response patterns of mechanoreceptive afferents from the monkey hand. Journal of Neurophysiology, 31, 301-334.

VerriLlo, R. T. (1966). Vibrotactile sensitivity and the frequency response of the Pacinian corpuscle. Psychonomic Science, 4, 135-136.

VERriLlo, R. T. (1968). A duplex mechanism of mechanoreception. In
D. Kenshalo (Ed.), The skin senses (pp. 139-159). Springfield, IL: Thomas.

Verrillo, R. T., Fraioli, A. J., \& Smith, R. L. (1969). Sensation magnitude of vibrotactile stimuli. Perception \& Psychophysics, 6, 366372.

VerriLlo, R. T., \& Gescheider, G. A. (1977). Effect of prior stimulation on vibrotactile thresholds. Sensory Processes, 1, 292-300.

WEISENBERGER, J. (1986). Sensitivity to amplitude-modulated vibrotactile signals. Journal of the Acoustical Society of America, 80, 1707-1715.

\section{NOTES}

1. Given that (1) RA threshold at $100 \mathrm{~Hz}$ is equivalent to that at $10 \mathrm{~Hz}$ (cf. Bolanowski et al., 1988), (2) a 10-Hz, 30-dB SL adapting stimulus increases RA thresholds by at least $10 \mathrm{~dB}$ (Bensmaïa and Hollins, 2000), and (3) unadapted thresholds at $10 \mathrm{~Hz}$ were found in the present study to be more than $13 \mathrm{~dB}$ higher than their counterparts at $100 \mathrm{~Hz}$, it can be concluded that adapted RA thresholds were at least $23 \mathrm{~dB}$ above PC threshold at $100 \mathrm{~Hz}$. Furthermore, this difference presumably increased at higher frequencies, since RA thresholds tend to increase with frequency beyond $100 \mathrm{~Hz}$, whereas PC thresholds drop dramatically (see Bolanowski et al., 1988); the RA system therefore contributed little to the perception of even the most intense stimuli. We thus feel confident that the perception of the vibratory stimuli was mediated predominantly if not exclusively, by the PC system.

2 . Both this and the following (spectral) model were also tested without incorporating Ekman's (1956) law. In both cases, the fit was substantially better when the difference in sensation magnitude was normalized by mean sensation magnitude (or by its counterpart in the spectral model). Furthermore, invoking Ekman's law is sensible, since a small difference in the pattern of activation elicited in the population of minichannels by two stimuli is going to be less salient if the overall activation produced by the stimuli is high than if it is low.

3 . The number of minichannels did not affect our results. A model with as few as 20 minichannels yielded an equivalent fit.

4. This latter probability is an overestimate, given that the model was fit to the scrambled data obtained from Stimulus Set 2 by adjusting all five parameters, rather than taking the parameter values optimized from Stimulus Set 1 and only varying $a_{400 \mathrm{~Hz}}$, as we did to obtain the test value $\rho=.88$.

5. In order to interpolate from Franzén's (1969) values, a logarithmic function was fit to his exponent estimates.

6 . Following the suggestion of an anonymous reviewer, another, related model was implemented that set the standard deviation of the Gaussians to $\alpha f+\beta f^{2}$, where both $\alpha$ and $\beta$ were free parameters (along with the exponents $a_{f}$ ). In this alternate model, the (linear) function relating the Weber fraction to the standard frequency was free to have a nonzero intercept. The model performed equivalently as the constant Weber fraction model ( $\rho=.89$ for both Data Sets 1 and 2), despite the fact that it had an additional degree of freedom. The outcome of this modeling effort thus favors the simpler model and lends support to its underlying assumption about the shape of the function relating frequency DL to standard frequency.

(Manuscript received September 12, 2003; revision accepted for publication August 30, 2004.) 